\title{
COMPRENDRE
}

\section{la mesure de taille de nanoparticules par diffusion dynamique de la lumière}

Maëlle MAINARD, David JACOB

Cordouan Technologies - Pessac maelle.mainard@cordouan-tech.com, david.jacob@cordouan-tech.com
Depuis leur invention dans les années 60, les lasers et leurs applications ont connu de spectaculaires développements scientifiques et industriels. En effet, grâce aux propriétés spectrales et spatiales uniques des lasers, l'étude de l'interaction matière-rayonnement a permis de sonder plus précisément et intimement la matière sous toutes ses formes. De nouvelles applications telles que la granulométrie laser ont ainsi vu le jour dès les années 70 et n'ont cessé de se développer depuis lors.
$\mathrm{P}$ arallèlement à ces développements technologiques et scientifiques autour des lasers, les chimistes et les physiciens ont commencé à s'intéresser aux nanoparticules et aux nanomatériaux, ainsi qu'à leurs propriétés remarquables. Encore simple curiosité de laboratoire il y a vingt ans, ces nanoparticules connaissent aujourd'hui un essor très impressionnant dans les laboratoires de recherche académiques et industriels. Les domaines adressés sont aussi variés que l'industrie pharmaceutique et la pétrochimie, en passant parl'agro-alimentaire, la biologie, ou encore les nouveaux matériaux; avec des applications telles que le traitement du cancer du sein, la mise au point de batteries électriques de nouvelle génération pour les systèmes embarqués, la vectorisation médicamenteuse, la détection de nano-polluant dans l'environnement, la fabrication de revêtements aux propriétés antiseptiques ou hydrophobes, les emballages intelligents pour l'agroalimentaire, ou même le traitement de l'eau... L'essor de ces nanomatériaux nécessite des moyens de caractérisation fiables, rapides et précis. Différentes techniques, directes ou indirectes (TEM, SAXS, AFM, etc.) sont aujourd'hui utilisées dans les laboratoires de recherche. Parmi elles, la technique optique de diffusion dynamique de la lumière (plus connue sous l'acronyme anglais DLS: dynamic light scattering) est certainement la plus répandue du fait de sa simplicité de mise en œuvre et de ses capacités de mesure. Cet article a pour objectif d'en décrire les grands principes et ses principales applications.

\section{La mesure de taille de namoparticules par DLS}

\section{Rappel sur les nanoparticules et le mouvement Brownien}

Les nanoparticules (NPs) sont des objets dont les dimensions sont comprises typiquement entre $1 \mathrm{~nm}\left(10^{-9} \mathrm{~m}\right)$ et $100 \mathrm{~nm}$. En suspension dans un milieu liquide, les forces d'Archimède et de gravité étant négligeables, les NPs sont principalement soumises aux chocs continus avec les molécules du liquide qui provoquent un mouvement désordonné et aléatoire des particules; il s'agit du mouvement Brownien, du nom du botaniste Robert Brown qui a pour la première fois observé ce mouvement sur des spores végétaux en 1827 (voir figure 1).
La première description mathématique de ce mouvement aléatoire est due à $\mathrm{L}$. Bachelier qui a démontré en 1901 que la valeur quadratique moyenne du déplacement d'une particule est proportionnelle au temps d'observation $t$. Le coefficient de proportionnalité noté $D$ est appelé coefficient de diffusion.

$$
\left\langle X^{2}(t)>\sim 2 D t\right.
$$

Il a fallu attendre 1905 et les travaux d'Albert Einstein pour avoir une première description physique du mouvement Brownien. Ainsi, à partir d'un modèle de sphères dures sans interaction et en utilisant une approche statistique, Einstein a établi une relation

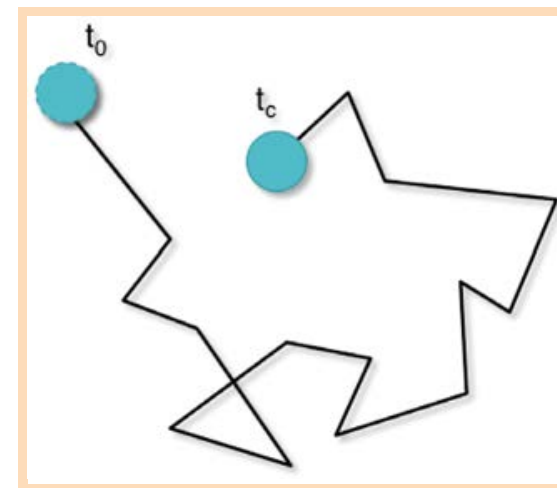

Représentation du mouvement brownien d'une particule entre $t_{0}$ et $t_{c}$. 


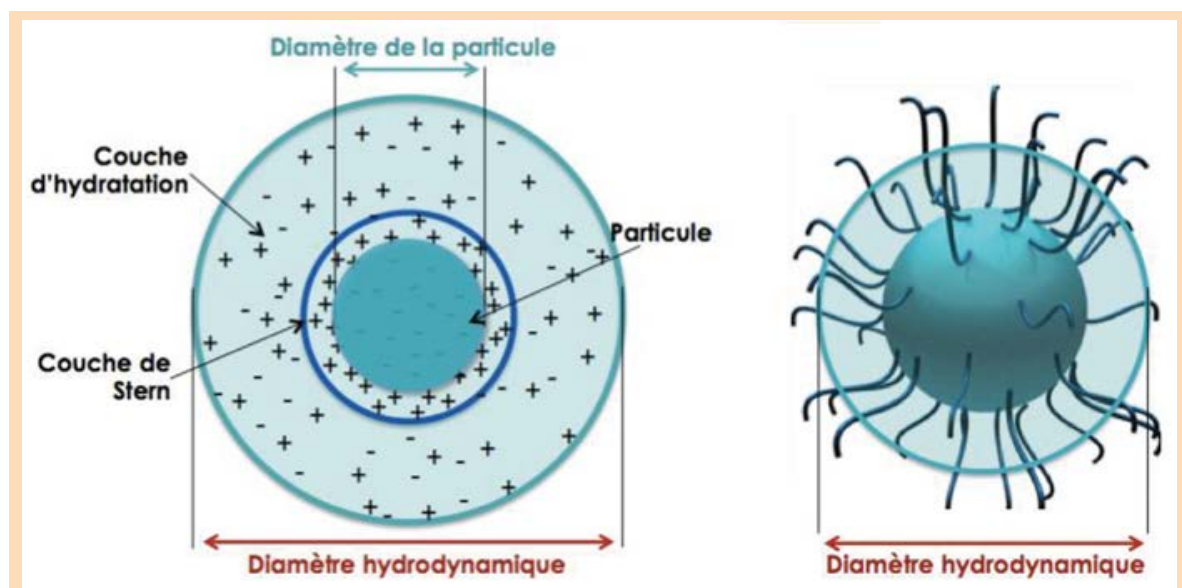

Figure 2. Représentation d'une particule avec sa couche d'hydratation constituant ensemble le diamètre hydrodynamique de la particule.

simple et directe entre le coefficient de diffusion $D$, le diamètre hydrodynamique des particules $\emptyset_{\mathrm{H}}$ et les propriétés du milieu liquide.

$$
D=\frac{k T}{3 \pi \mu \emptyset_{\mathrm{H}}}
$$

Dans la relation (2), $\mu$ est la viscosité du solvant, $T$ la température du solvant, $k$ la constante de Boltzmann et $\emptyset_{\mathrm{H}}$ le diamètre hydrodynamique de la particule qui prend en compte le diamètre de la particule et sa couche dite d'hydratation, comme représenté dans la figure 2. Connaissant la température et la viscosité du milieu liquide, on voit donc qu'une mesure du coefficient de diffusion $D$ permet de déterminer $\emptyset_{\mathrm{H}}$ selon l'équation de Stokes-Einstein (3):

$$
\emptyset_{\mathrm{H}}=\frac{k T}{3 \pi \mu D}
$$

Comme nous allons le voir dans le paragraphe suivant, c'est sur ce principe physique fondamental que repose la mesure de taille de nanoparticules par DLS.

\section{La technique de mesure par diffusion dynamique de la lumière}

La DLS est une technique optique qui utilise donc le mouvement Brownien comme une «signature» de la taille des particules en suspension par la mesure de leur coefficient de diffusion $D$. Un dispositif de DLS classique est représenté sur le schéma de principe ci-dessous en figure 3: une source laser polarisée linéairement éclaire à une longueur d'onde $\lambda$ (typiquement $633 \mathrm{~nm}$ ) l'échantillon contenant les nanoparticules à mesurer; la lumière diffusée par les nanoparticules en mouvement Brownien est ensuite collectée à un angle de diffusion $\theta$ connu (typiquement $90^{\circ}$ par rapport au faisceau incident) grâce à un détecteur à comptage de photons de haute sensibilité (photodiode à avalanche - APD ou tube photo multiplicateur - TPM). Cette lumière diffusée créée donc au niveau du

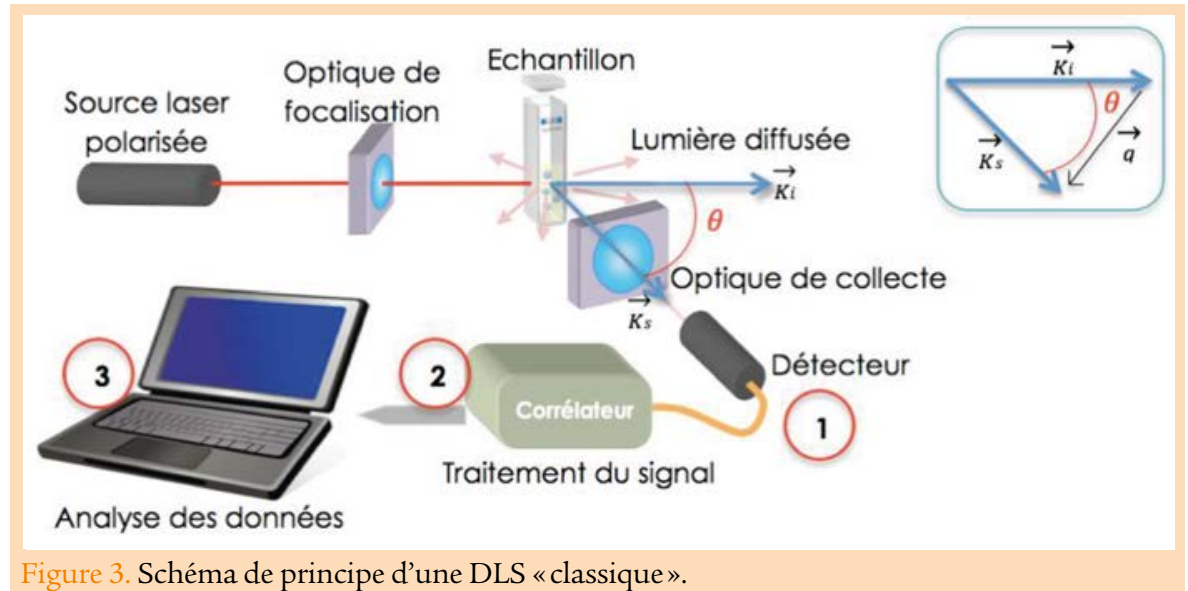

Figure 3. Schéma de principe d'une DLS «classique».

\section{small components MASSIVE IMPACT}

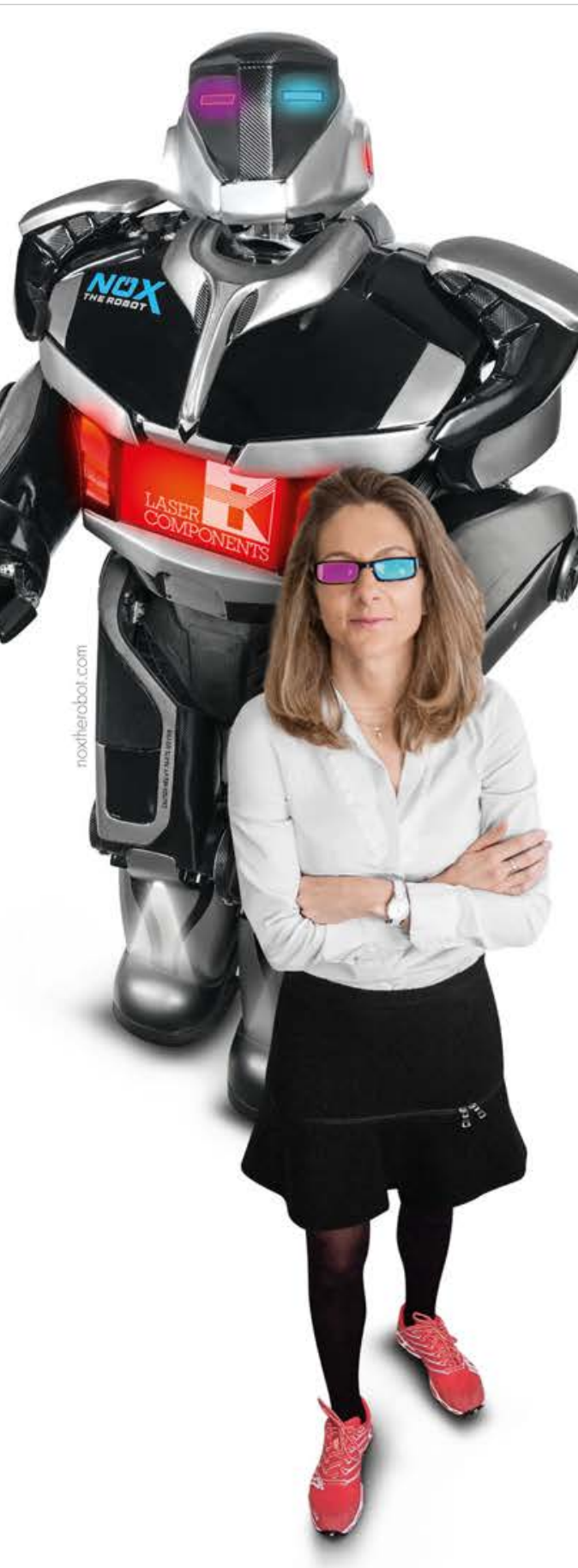

Optical Filters

- Bandpass Filters

- Short-Pass / Long-Pass Filters

- Fluorescence Filters

- IR Filters 
détecteur l'équivalent d'une figure de speckle ponctuelle dont les fluctuations dynamiques sont corrélées aux propriétés du mouvement Brownien.

La figure 4 ci-dessous illustre les trois étapes d'une mesures de DLS : le détecteur convertit les fluctuations d'intensité diffusée dues au mouvement Brownien des particules (1) en un signal électronique qui est ensuite traité numériquement par une carte électronique dédiée (l'auto-corrélateur), pour générer une fonction d'autocorrélation (2) [1] en intensité.

On peut montrer [1] que la fonction d'autocorrélation s'écrit sous la forme d'une (ou plusieurs) exponentielle(s) décroissante(s) donnée(s) parl'équation (4).

$$
G_{2}(\tau)=A+\beta \exp \left(-2 q^{2} D \tau\right)
$$

où $\tau$ est l'intervalle de temps, $\beta$ une constante liée à la configuration de mesure (facteur d'instrument) et $q$ le vecteur d'onde de diffusion donné par $q=\frac{4 \pi \mathrm{n}_{0}}{\lambda} \sin (\theta / 2) ;$ On notera dans l'équation (4) la présence du coefficient de diffusion $\mathrm{D}$ de l'équation de Stokes Einstein.

Dans une dernière étape de traitement (3), des algorithmes d'inversion permettent de «fitter» le corrélogramme et d'extraire de façon précise les distributions de taille (diamètre hydrodynamique) ainsi que l'indice de polydispersité (PDI) des particules. Il existe de nombreux algorithmes d'inversion dont le plus utilisé demeure Cumulants. Cet algorithme standardisé est basé sur un modèle mono-exponentiel qui permet de déterminer un diamètre hydrodynamique moyen $\left(Z_{\text {average }}\right)$ et une largeur de distribution ou indice de polydispersité (PDI). Cumulants est pertinent pour des échantillons monomodaux, i.e. comprenant une seule population de taille. Dans le cas d'échantillons complexes polymodaux présentant plusieurs populations (cas par exemple des échantillons en présence d'agrégats, ou lors des phases de synthèse de NPs), l'algorithme des Cumulants ne permet plus de donner une représentation fidèle de l'échantillon. On utilise alors des algorithmes mathématiques plus élaborés tels que CONTIN, NNLS (non negative least square), SBL (sparse Baysian learning), maximum entropy, etc. Ces algorithmes élaborés à base de calculs matriciels, de régularisateurs et inversion de Laplace allient des méthodes statistiques et analytiques, permettant à la fois de trouver plusieurs tailles dans un échantillon polydisperse mais également de donner la distribution autour de ces tailles.

Traditionnellement, un dispositif de mesure de taille de nanoparticules par DLS est un instrument dit de paillasse (voir figure 5). L'échantillon est généralement versé dans une cuvette de section carrée $(10 \times 10 \mathrm{~mm})$ en plastique transparent ou en quartz. Cette cuvette est alors placée dans un réceptacle contrôlé en température à l'intérieur de l'appareil. La détection de la lumière diffusée peut se faire selon différents angles. Historiquement, la configuration à $90^{\circ}$ est la plus répandue. L'une des limitations majeures de cette configuration est l'impossibilité de mesurer des milieux concentrés du fait de la turbidité du milieu et des effets de diffusions multiples qui biaisent la mesure. L'utilisateur est donc obligé de fortement diluer son échantillon pour le rendre mesurable.

Pour pallier à cette limitation, une alternative consiste à mesurer l'échantillon en rétrodiffusion et en couche mince. Ainsi un instrument commercial propose un dispositif avec une cellule de mesure intégrée composée d'un prisme en silice et d'un couvercle de cellule comportant un système de contrôle de l'épaisseur du film liquide à analyser. Ce système de contrôle présente ainsi deux positions (dual thickness controller, DTC): une position haute (épaisseur $1 \mathrm{~mm}$ ) pour la mesure des milieux dilués, et une position basse (épaisseur $100 \mu \mathrm{m}$ ) pour les milieux concentrés (voir figure 5). Dans cette configuration, il suffit de verser une goutte (typiquement $50 \mu \mathrm{L}$ ) de la suspension à caractériser sur la face supérieure du prisme, d'ajuster la position du DTC selon la concentration de l'échantillon et de lancer la mesure. Le DTC permet ainsi, sans dilution préalable, d'étendre la gamme de mesure en concentration d'environ deux décades, de $10^{-4} \%$ jusque $40 \%$ en volume, par rapportà une mesure classique en cuve à $90^{\circ}$.

\section{Les avantages de la DLS «traditionnelle» de paillasse}

LaDLS traditionnelle de paillasse, en cuve ou film mince, offre de nombreux avantages qui en ont fait le succès depuis plus de trente ans dans les laboratoires de recherche. Cette méthode offre une impressionnante gamme de mesure de taille de particules sur plus de 4 décades, de 1 nmà $10 \mu \mathrm{m}$. La DLS est une technique mature et désormais normalisée au niveau international (ISO 13321 et ISO 22412). C'est par ailleurs une technique relativement simple, robuste et facileà mettre en cuvre (une mesure de distribution de taille ne prend que quelques minutes), avec des instruments dont l'encombrement est raisonnable pour un usage sur paillasse et ne nécessitant pas de servitudes de fonctionnement particulières. En outrele prix des instruments commerciaux, entre $25 \mathrm{k} €$ et $70 \mathrm{k} €$ selon les fournisseurs et les options proposées, rendent la DLS très attractive en complément ou alternative

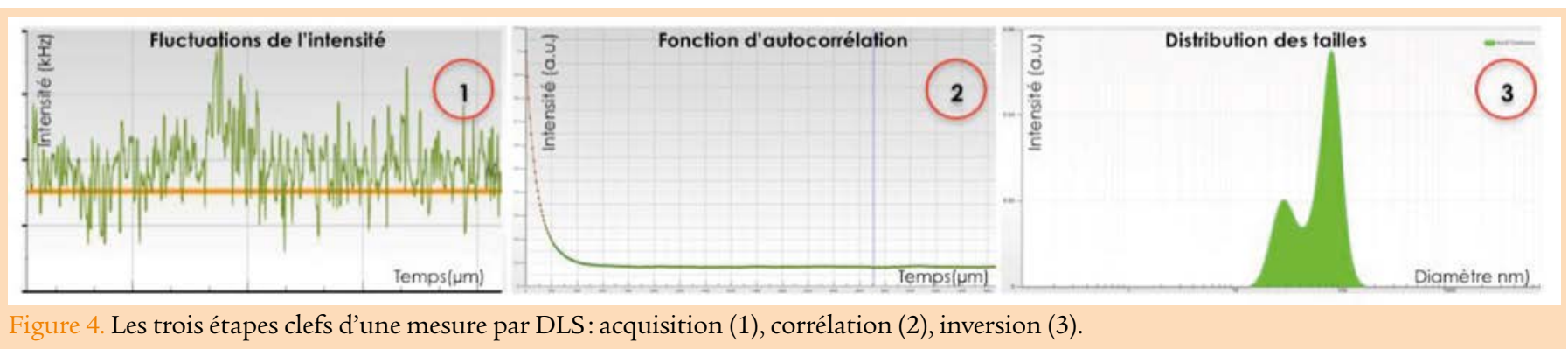




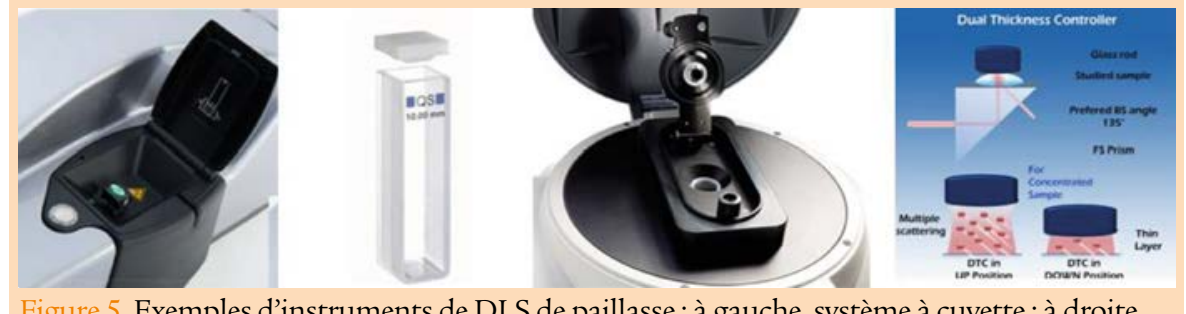

Figure 5. Exemples d'instruments de DLS de paillasse : à gauche, système à cuvette; à droite, système avec cellule de mesure intégrée et mesure en film mince d'épaisseur contrôlable (DTC).

à d'autres techniques de caractérisation plus onéreuses et/ou plus encombrantes telles que le SAXS, l'AFM, le TEM, etc.

\section{Les avancées récentes de la DLS}

Si la DLS traditionnelle permet de répondre à la plupart des besoins de mesure par prélèvement en laboratoire, ces instruments de paillasse ne sont plus adaptés lorsqu'il s'agit de faire des mesures in situ, sans prélèvement, et/ou en environnement contraint (autoclave, enceinte hermétique, boite à gant, four de synthèse, etc.).

Tirée par cette demande émergente des laboratoires de recherche et des industriels pour le suivi de procédé, la DLS connait aujourd'hui de nouveaux développements reposant sur un changement de paradigme expérimental que l'on peut résumer par: «s'il est impossible d'amener l'échantillon à la mesure, il faut amenerla mesure à l'échantillon ou au procédé». C'est sur ce nouveau principe qu'un dispositif tel que la sonde de mesure in situ et déportée par fibre optique a récemment vu le jour au niveau commercial. Le principe de cette nouvelle sonde et des exemples d'applications concrets sont présentés dans la section suivante.

\section{La technique de la DLS déportée par fibre optique}

Grâce à une première fibre optique et un système de lentilles adaptées, il devient possible d'acheminer et de focaliser la source laser à l'endroit où se trouve l'échantillon. La lumière diffusée par les particules est alors collectée par un système de lentilles puis acheminée jusqu'au détecteur par une deuxième fibre optique, comme illustré dans la figure 6a. Ces éléments constituent ainsi le corps de la sonde optique déportée qui est connectée par un ombilical contenant les deux fibres optiques à une unité centrale. Cette dernière comprend la source laser, le détecteur, le corrélateur, les alimentations et modules de communication avec un PC ainsi qu'un laser d'alignement. L'angle de détection pour la DLS déportée est de $170^{\circ}$. Cette configuration permet en effet, selon la théorie de Mie, de détecter les petites particules, sans que celles-ci ne soient masquées par la diffusion des grosses particules de l'échantillon.

\section{Les avantages de la DLS déportée}

Ce système de mesure de taille de NPs déportée par fibre optique demeure robuste, rapide et viable; ce à quoi s'ajoute également, grâce à la petite taille de la sonde de mesure et à la longueur ajustable de la fibre optique connectée à l'unité centrale, une grande facilité d'intégration, donnant accès à des analyses sans prélèvement. Cette mesure déportée est donc in situ et sans contact avec l'échantillon étudié, puisque la distance de travail est d'environ 7 à $8 \mathrm{~cm}$, il n'y a donc aucun risque de contamination croisée des échantillons. Pour une plus grande facilité de positionnement, la tête comprend également un laser d'alignement permettant de détecter la zone de mesure et de positionner l'échantillon de façon optimale. Ce système de mesure déportée permet par ailleurs d'observer en temps réel la cinétique de synthèses au sein de réacteurs.

\section{Les applications rendues possibles grâce à cette avancée}

La mesure de taille de NPs par DLS déportée par fibre optique ouvre le champ à certaines applications jusqu'alors

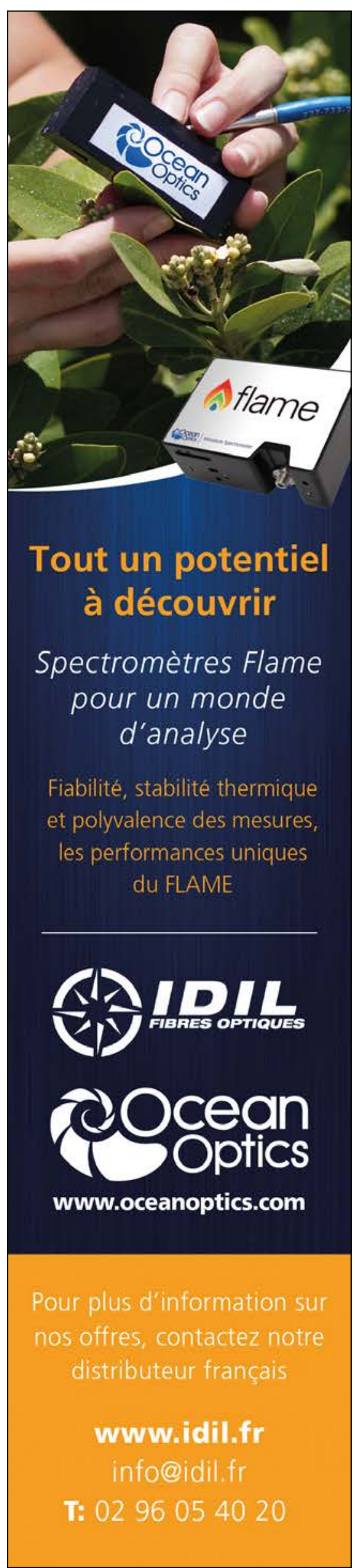




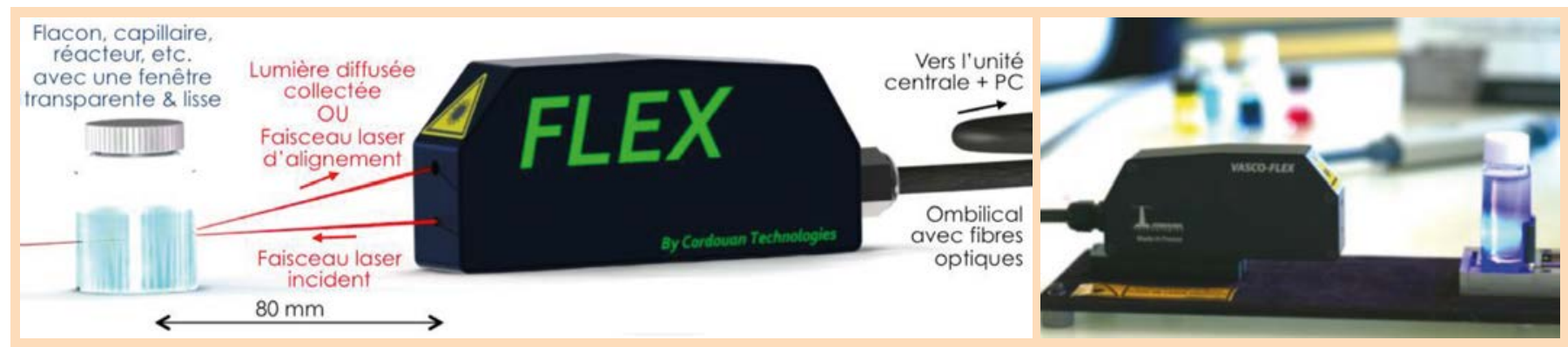

Figure 6. (a) Schéma de principe de la sonde DLS déportée par fibre optique. (b) Exemple de réalisation d'un dispositif commercial.

impossibles. Cela a notamment permis: (1) de travailler dans des milieux confinés et/ou difficiles (haute température, haute pression, radioactivité, gaz inerte, etc.); (2) de coupler la DLS à d'autres technologies pour une caractérisation plus complète des nanoparticules et (3) de suivre «en live» des synthèses pour lesquelles le prélèvement est impossible sans perturber la réaction.

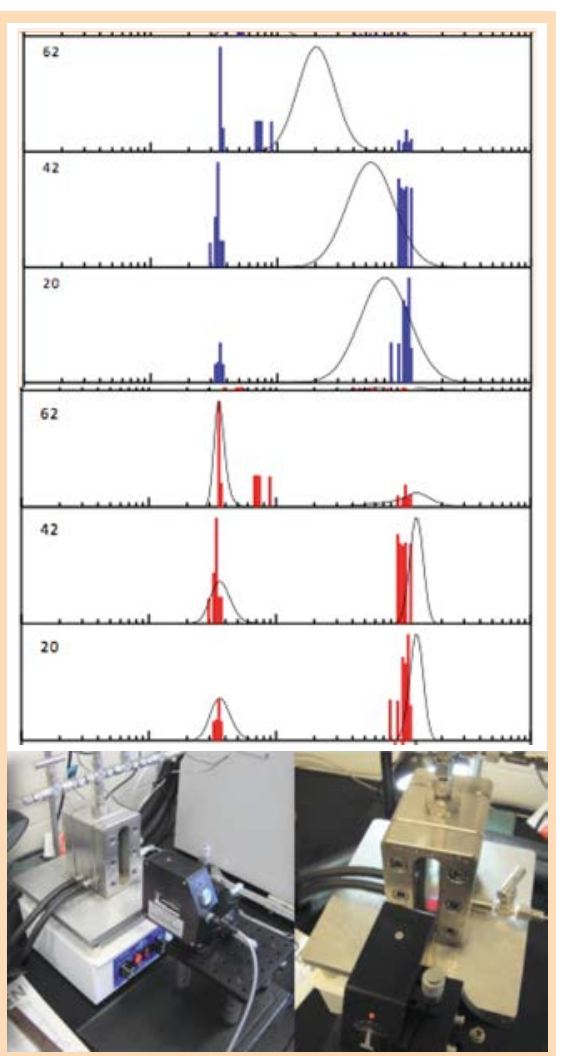

Figure $7:$ (a) Exemple d'applications rendues possibles par la DLS déportée: étude de la cinétique de la synthèse de polymères au sein d'un réacteur à $\mathrm{CO}_{2}$ supercritique. (b) Évolution de la distribution des tailles de nanoparticules dans le temps (superposition et comparaison des différents algorithmes: Padé Laplace (haut, bleu) vs. Cumulants (haut, trait fin) et SBL (bas, rouge) vs. Cumulants (bas, trait fin)).
Nous pouvons citer ici plusieurs exemples d'applications concrètes: (1) l'observation de synthèse en réacteur à $\mathrm{CO}_{2}$ supercritique (voir figures $7 a$ et $7 b$ ) ou encore le développement d'une méthode de traitement de cellules cancéreuses par hyperthermie magnétique (car la mesure par DLS déportée est insensible aux champs magnétiques [2]); (2) le couplage au SAXS [3] permettant de mesurer sur une plus large gamme de tailles (de quelques Å à quelques $\mu \mathrm{m}$ ) et d'obtenir des informations sur la structure et sur l'agrégation des particules en simultané; (3) l'étude du procédé de cristallisation d'ibuprofene ou bien la fabrication de quantum dots. Mais cette nouvelle technologie s'adapte également au monde industriel. En effet, la mesure par DLS déportée permet un contrôle de procédés et un contrôle qualité tout au long des lignes de production industrielles (notamment, en pharmacie).

En outre, l'essor de l'utilisation des nanoparticules dans un nombre croissant de domaines pose également la question de l'impact environnemental et sanitaire. Une fois encore, la DLS déportée va être un élément central dans les systèmes complexes d'identification de ces éléments dans les matrices naturelles, et permettra des avancées scientifiques dans la compréhension des effets éco-toxicologiques [4].

\section{Conclusion}

Après plus de 40 ans d'existence et d'évolutions continues, la DLS est devenue aujourd'hui une technique de caractérisation de suspensions colloïdales mature et reconnue dans les laboratoires de recherche. En effet, sa simplicité d'usage, sa rapidité de mesure et son prix d'accès modéré ont largement contribué à en faire un outil de mesure incontournable pour caractériser de manière statistique des distributions de taille de nanoparticules en suspension, en complément d'autres techniques de caractérisation.

La DLS connait aujourd'hui de nouveaux développements tels que la mesure sans contact déportée par fibre optique. Ils permettent d'envisager son utilisation en dehors du monde usuel des laboratoires, et d'ouvrir de nouveaux débouchés applicatifs comme le couplage instrumental, le suivi de procédés de synthèse en temps réel, la mesure en environnement contraint, etc. En pleine maturité, la DLS a encore de beaux jours devant elle dans les laboratoires mais aussi pour des applications industrielles.

\section{REFÉRENCES}

[1] Dynamic Light Scattering with Applications to Chemistry, Biology, and Physics, B.J. Berne, R. Pecora, 1976, Dover edition

[2] Thermosensitive polymer-grafted iron oxide nanoparticles studied by in situ dynamic light backscattering under magnetic hyperthermia, G. Hemery, E. Garanger, S. Lecommandoux, A.D. Wong, E.R. Gillies, B. Pedrono, T. Bayle, D. Jacob, O. Sandre, 2015

[3] Combining SAXS and DLS for simultaneous measurements and time-resolved monitoring of nanoparticle synthesis, A. Schwamberger, B. De Roo, D. Jacob, L. Dillemans, L. Bruegemann, J.W. Seo, J.P. Locquet

[4] Marine Plastic litters: the unanalysed nano-fraction, J. Gigault, B. Pedrono, B. Maxit, A. ter Halle, 2016, Royal Society of Chemistry, DOI: 10.1039/ C6EN00008H 